\title{
Les contraintes socio-environnementales du manque d'eau potable au sein des villages Avikam du cordon littoral de Grand-Lahou (Côte d'Ivoire)
}

\author{
Dadi Reine Prisca \\ Doctorante en Sociologie de l'Environnement \\ Université Félix Houphouët-Boigny, Côte d'Ivoire
}

Doi:10.19044/esj.2021.v17n14p70

Submitted: 06 February 2021

Accepted: 09 March 2021

Published: 30 April 2021
Copyright 2021 Author(s)

Under Creative Commons BY-NC-ND

4.0 OPEN ACCESS

Cite As:

Prisca D.R. (2021). Les contraintes socio-environnementales du manque d'eau potable au sein des villages Avikam du cordon littoral de Grand-Lahou (Côte d'Ivoire). European Scientific Journal, ESJ, 17(14), 70. https://doi.org/10.19044/esj.2021.v17n14p70

\section{Resume}

L'objectif du présent article est d'analyser les contraintes socioenvironnementales qui font obstacle à l'accès à l'eau potable au sein des villages Avikam du cordon littoral de Grand-Lahou. Les contraintes ici représentent un ensemble de réalités naturelles, économiques, organisationnelles, matérielles et morales qui entravent le processus d'acquisition en eau potable. Cet article, s'inscrivant dans une approche qualitative, a mobilisé des techniques de recueils de données telles que : l'étude documentaire, l'entretien semi-directif, l'observation et l'échantillonnage. La méthode par choix raisonné a permis de sélectionner les 40 personnes enquêtées. Il ressort de cette étude que les contraintes socioenvironnementales qui entravent l'accès à l'eau potable au sein des villages Avikam du cordon littoral de Grand-Lahou sont : le refus de l'État à investir à perte, l'attachement des populations à leur patrimoine ancestral, l'enclavement de l'espace, l'érosion côtière, l'absence de voie terrestre et la proximité des villages aux sources d'eau naturelles.

Mots-clés : Eau potable, contraintes socio-environnementales, milieu rural, sociologie de l'environnement 


\title{
The Socio-Environmental Constraints of the Lack of Drinking Water in the Avikam Villages of the Coastal Barrier of Grand-Lahou (Ivory Coast)
}

\author{
Dadi Reine Prisca \\ Doctorante en Sociologie de l'Environnement \\ Université Félix Houphouët-Boigny, Côte d'Ivoire
}

\begin{abstract}
The objective of this article is to analyze the socio-environmental constraints that obstruct access to drinking water in the Avikam villages of the coastal barrier of Grand-Lahou. The constraints here represent a set of natural, economic, organizational, material and moral realities that hinder the process of acquiring drinking water. While subscribing to a qualitative approach, data collection techniques such as: documentary study, semi-structure interview, observation and sampling were used. The reasoned choice method made it possible to select the 40 resource people surveyed. It emerges from this study that the socio-environmental constraints which hinder access to drinking water in the Avikam villages of the coastal barrier of Grand-Lahou are, the refusal of the state to invest at a loss, the attachment populations to their ancestral heritage, the isolation of space, coastal erosion, lack of land and the proximity of villages to natural water sources.
\end{abstract}

Keywords: Drinking water, socio- environmental constraints, rural environment, sociology of the environment

\section{Introduction}

L'eau potable est une ressource essentielle dans le développement humain, économique et social de toute société. Vu l'intérêt que revêt cette ressource, 1'Organisation des Nations Unies (ONU) s'est engagée à travers le sixième $\left(6^{\text {ème }}\right)$ objectif du développement durable (ODD) à garantir l'accès de tous à l'eau et à l'assainissement et assurer une gestion durable des ressources en eau d'ici 2030 (ODD, 2015). À partir de cette conférence, la question de l'accès à l'eau potable va s'imposer comme une priorité pour tous les États du monde.

La Côte d'Ivoire, à l'instar des autres pays d'Afrique subsaharienne, avait déjà inscrit la question de l'approvisionnement en eau potable dans ses plans d'actions de développement. Cette insertion des questions hydrauliques a permis de structurer les besoins hydriques en trois sous-secteurs que sont : l'hydraulique urbaine (HU), l'hydraulique villageoise (HV) et l'hydraulique 
villageoise améliorée (HVA) [Diabagaté et al, 2016]. Ainsi, pour s'inscrire dans la vision du sixième ( $\left.6^{\text {ème}}\right)$ objectif des ODD, le gouvernement ivoirien a mis en place plusieurs programmes dont le programme d'hydraulique et d'assainissement pour le millénaire (PHAM), le programme «eau pour tous», le programme social du gouvernement etc. Ces programmes visent d'une part, à améliorer la desserte en eau de toutes les populations vivant sur le territoire ivoirien et d'autre part, à porter le taux d'accès à l'eau potable à $95 \%$ jusqu'en 2030 (ONEP, 2017). Le PHAM a permis la satisfaction hydrique de plus de cent cinquante mille (150 000) villageois des régions de l'Ouest ivoirien, la mise à niveau de treize (13) systèmes d'amélioration en eau potable (remplacement de canalisations, construction de nouvelles bornes fontaines etc.) et la réalisation de vingt-neuf (29) nouveaux systèmes d'Adduction en Eau Potable [AEP] (équipements et branchements électriques des forages, pose de canalisations etc.) [Marteau, 2017]. Quant aux autres programmes, ils ont permis au district d'Abidjan d'augmenter sa production en eau potable de $350000 \mathrm{~m}^{3} / \mathrm{j}$ à $640000 \mathrm{~m}^{3} / \mathrm{j}$ et aux chefs-lieux des régions de passer de $79655 \mathrm{~m}^{3} / \mathrm{j}$ à $131566 \mathrm{~m}^{3} / \mathrm{j}$ soit un apport de $51911 \mathrm{~m}^{3}$. Quant aux villes de l'intérieur du pays, 44 localités ont bénéficié du renforcement de leur production en eau, 22 localités de l'extension de leurs réseaux hydrauliques, 25 localités de la mise en place d'Adduction en Eau Potable (AEP) et la réalisation de l'AEP de 13 nouvelles localités ont été finalisées. Le milieu rural a également bénéficié de la réhabilitation de 5000 pompes à motricité humaine (PMH) et de la maintenance courante de $16693 \mathrm{PMH}$. Au total, 21000 pompes à motricité humaine ont été construites dans les zones rurales (Ministère de 1'hydraulique, 2019). L'ensemble de ces réalisations susmentionnées a permis au pays d'obtenir un taux de couverture nationale en eau potable estimé à plus de $80 \%$ en fin 2018 (Tchagba, 2019). Le département de Grand-Lahou n'est pas resté en marge de ces programmes qui ont permis, de résorber les problèmes d'accès à l'eau potable par l'installation de plusieurs ouvrages hydrauliques que sont : un forage d'une grande capacité au sein de la commune de Grand-Lahou, des châteaux dans les souspréfectures du département (un château par sous-préfecture) et des [HVA, HV]. dans plusieurs villages (Nogbou, 2018). Nonobstant les réalisations faites sur l'ensemble du territoire ivoirien et au sein du département de GrandLahou, certains milieux ruraux comme l'ensemble des dix (10) villages du cordon littoral du département de Grand-Lahou présentent toujours une vulnérabilité hydrique. Cette vulnérabilité est perceptible par l'inexistence d'ouvrages hydrauliques (HV, HVA) au sein de tous les villages et un nombre important de points d'eau traditionnels (puits, marigots) à ciel ouvert. Au regard de la situation hydrique susmentionnée, il est opportun d'essayer de comprendre pourquoi les villages du cordon n'ont pas été intégrés par les pouvoirs publics dans les différents projets hydrauliques réalisés. En d'autres 
termes, quelles sont les contraintes socio-environnementales qui entravent la réalisation d'ouvrages hydrauliques au sein des villages du cordon littoral de Grand-Lahou ?

L'objectif du présent article est d'analyser les contraintes socioenvironnementales qui entravent l'accès à l'eau potable au sein des villages Avikam du cordon littoral de Grand-Lahou.

\section{Méthodologie}

Les données utilisées ont été collectées de février 2017 à juin 2018 à Abidjan, à Dabou, à Grand-Lahou et au sein de quatre (4) villages Avikam du cordon littoral de Grand-Lahou que sont : Allèkèdon, Zagbalébé, Kokou et Lahou- Kpanda.

\section{1-1. Zone de l'étude}

Le département de Grand-Lahou est limité au Nord par le département de Tiassalé et celui de Divo, au Sud par l'Océan Atlantique, à l'Est par les départements de Jacqueville, de Dabou et de Sikensi, à l'Ouest par les départements de Guitry et de Fresco.

Cette étude s'est précisément déroulée au sein de quatre (4) villages choisis sur les dix (10) villages que compte le cordon littoral du département de Grand-Lahou ce sont : Allèkèdon, Zagbalébé, Kokou et Lahou-Kpanda (Carte 1).

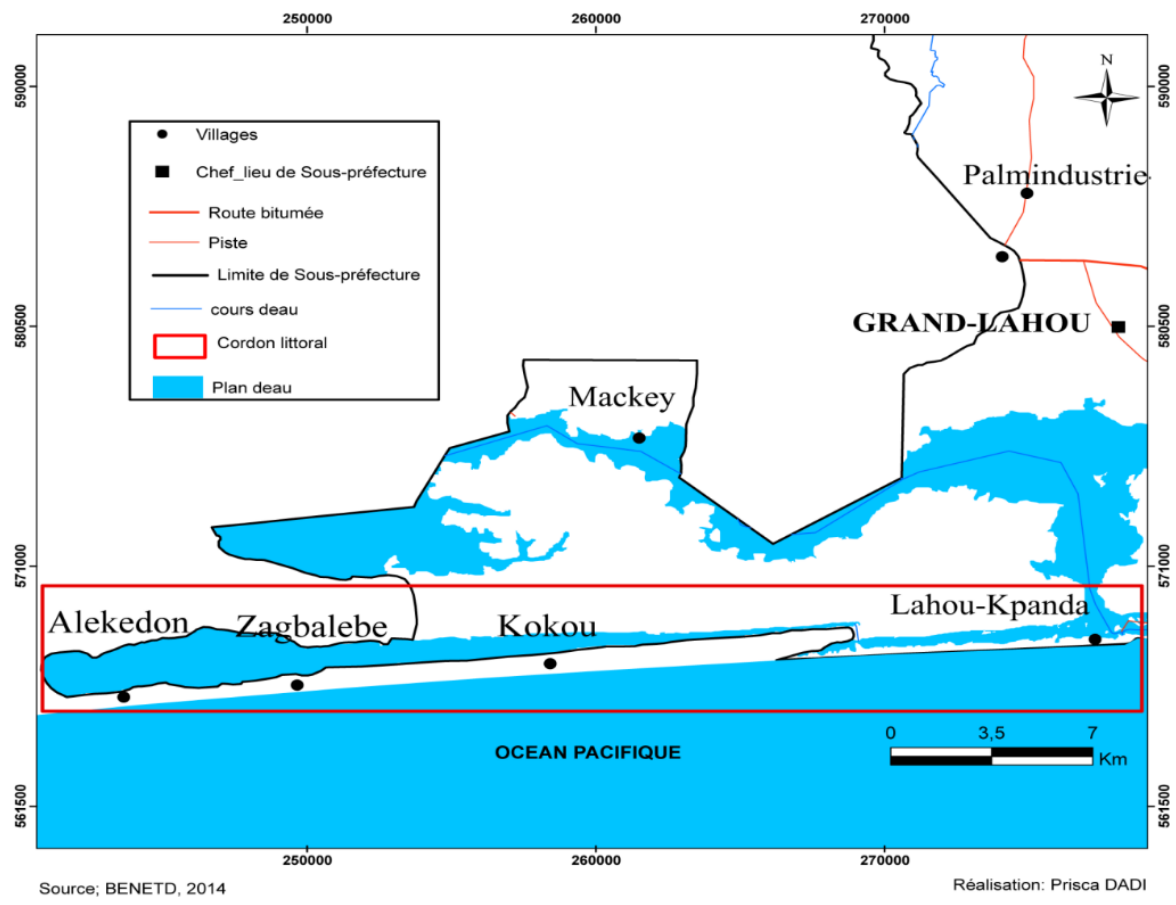

Figure 1 : Carte de localisation des 4 villages du cordon littoral Grand-Lahou 


\section{1-2. Techniques de collectes de données}

Le présent travail s'est appuyé sur trois techniques de collecte de données que sont : la recherche documentaire, l'observation et l'entretien.

La recherche documentaire a permis de saisir le phénomène du manque d'eau potable sur ses aspects social, économique, environnemental et sanitaire par l'exploration de la littérature en rapport avec la thématique en étude et à faire l'état des différents aspects ayant déjà été abordés par d'autres chercheurs.

L'observation a consisté à répertorier l'ensemble des points d'eau traditionnels (puits, marigots) existants dans chaque village. De cette observation, il ressort que les villages d'Allèkèdon, de Zagbalébé et de Kokou avec des populations estimées respectivement à 377,518 et 1628 personnes (INS, 2014), ne disposent que d'un seul puits traditionnel par village. Par contre, à Lahou-Kpanda chaque ménage possède un point d'eau traditionnel. Les points d'eau des villages du cordon littoral sont tous à ciel ouvert, positionnés pour la plupart à proximité des lagunes, avec des alentours humides, insalubres et distants de $500 \mathrm{~m}$ à $1 \mathrm{~km}$ des villages. Ensuite, l'eau contenue dans ces ouvrages traditionnels a une coloration variable (jaunâtre, rougeâtre, verdâtre, noirâtre, marron). Enfin, tous les points d'eau ne subissent aucun traitement tel que la chloration qui consiste à utiliser des produits chlorés (javel, pastille de chlore, les comprimés) afin d'éliminer tous les microorganismes avant toute consommation.

Quant à l'entretien, il a été mené selon la technique d'interview-centrée avec les parties prenantes. Il s'agit de la Société de Distribution d'Eau de Côte d'Ivoire (SODECI), de l'Office National de l'Eau Potable (ONEP), des collectivités territoriales (la Direction régionale de l'Hydraulique Humaine [DRHH], le conseil régional des Grands ponts, la préfecture de Grand-Lahou, les sous-préfectures et la mairie de Grand-Lahou), des institutions internationales (la Banque Africaine de Développement [BAD], l'Organisation Mondiale de la Santé [OMS], le Fonds des Nations Unies pour l'Enfance [UNICEF] ) et les personnes ressources des quatre (4) villages (les chefs de villages, les notables, les propriétaires terriens et les sages) afin de recueillir des informations sur leur niveau d'implication et de responsabilité dans l'approvisionnement en eau potable. L'entretien s'est fait par thématiques; il a été conduit au tour de trois axes :

- l'état des lieux de l'eau potable en milieu rural ivoirien et sur le cordon littoral de Grand-Lahou ;

- les contraintes socio-environnementales qui entravent l'extension du réseau hydraulique jusqu'au sein du cordon littoral ;

- les conséquences socio-environnementales et sanitaires qui en découlent. 


\section{1-3. Technique d'échantillonnage}

La technique d'échantillonnage adoptée est l'échantillonnage par choix raisonné. Cette technique consiste pour le chercheur, à sélectionner une partie représentative de la population cible qui sont des personnes ressources. Cet échantillonnage prend en compte les personnes-ressources des structures internationales, nationales et rurales. La répartition de l'échantillon s'est faite de la manière suivante: dix-huit (18) personnes-ressources au sein des structures nationales et internationales et vingt-deux (22) responsables au niveau des villages, soit un total de 40 personnes interrogées.

\section{1-4. Traitement des données}

Le traitement des données a débuté par le dépouillement manuel, qui a consisté à regrouper les réponses identiques se rapportant à un même caractère afin de rendre possible la description, l'analyse et l'explication de la problématique de l'accès à l'eau potable au sein des villages du cordon littoral de Grand-Lahou. La méthode d'analyse des données mobilisées par ce travail de recherche est l'analyse du contenu qui est «un ensemble de techniques de recherche permettant de décrire tout le contenu d'une communication en vue d'en déduire de manière logique des connaissances sur l'émetteur du message ou sur l'environnement" (Albarello et al, 1995). Cette méthode a permis d'analyser le contenu du discours des enquêtés sur les contraintes qui entravent l'accès à l'eau potable au sein des différents villages.

\section{Résultats}

Les résultats de cette étude révèlent l'existence de deux catégories de contraintes que sont: les contraintes sociales et les contraintes environnementales.

\section{2-1. les contraintes sociales du manque d'eau potable au sein des villages du cordon littoral de Grand-Lahou}

Cette catégorie est constituée de deux sous-contraintes à savoir, le refus de l'État d'invertir à perte et l'attachement des populations à leur patrimoine ancestral.

\section{2-1-1. Le refus de l'État d'investir à perte}

En Côte d'Ivoire, les projets d'accès à l'eau potable et à l'assainissement ont été initiés en faveur de tous les milieux ruraux depuis les années 1970. Ainsi, lors des échanges avec les responsables administratifs sur les logiques sociales qui pouvaient expliquer le manque d'eau potable au sein des villages du cordon littoral de Grand-Lahou jusqu'à présent, un enquêté a affirmé. : "Chère madame, en Côte d'Ivoire c'est l'État qui décide de quelle localité doit être prise en compte dans un projet en fonction de leur intérêt et 
ceux des partenaires au développement qui les accompagnent dans la réalisation desdits projets. Concernant le cordon, c'est un espace que l'érosion ronge progressivement depuis les années 1970 et qui va disparaître si aucune solution n'est trouvée. D'ailleurs, les projets planifiés n'ont souvent jamais tous abouti parce qu'ils nécessitent de gros moyens qui dépassent parfois le budget à eux alloué. Vu les dégâts de l'érosion et les menaces qui s'amplifient d'années en années c'est très compliqué ». De cette déclaration, on s'aperçoit que l'idéologie selon laquelle « le cordon va disparaître » qui s'est transmise d'une administration à une autre depuis 1970 à nos jours, dissimule le refus d'investir à perte de la part de l'État ivoirien et ses partenaires au développement. En effet, l'érosion manifeste sur le cordon littoral peut occasionner des investissements à perte en raison des dégradations inhérentes à la corrosion et à la forte brise de mer qui détruit tout sur son passage. Cette réalité a entraîné la marginalisation, voire l'exclusion du cordon littoral de presque tous les projets qui ont trait au développement (l'eau potable, l'électrification et l'équipement sanitaire) par les pouvoirs publics malgré les répercussions socio-économiques et sanitaires néfastes auxquelles ces populations peuvent-être exposées.

On peut retenir que, l'idéologie de la disparition du cordon transmise d'une administration à une autre depuis plusieurs décennies a entraîné le refus des structures étatiques d'investir à perte.

\section{2-1-2. L'attachement des populations à leur patrimoine ancestral}

Le cordon littoral de Grand-Lahou est un espace qui abritait l'ancienne ville historique et coloniale de Grand-Lahou crée vers 1910 sur la base d'un village Avikam (Hamaji, 2016). Avec les menaces de l'érosion, la ville a été déplacée dès 1971 et les autorités administratives ont demandé aux populations des villages du cordon littoral de migrer sur un autre site pour éviter les impacts de l'érosion côtière afin de bénéficier d'infrastructures modernes. À ce sujet, un enquêté dit ceci : «On n'a pas besoin de nous demander d'abandonner nos origines pour des infrastructures qu'on n'est même pas sûr d'avoir. Il y a des villages qui sont de l'autre côté mais qui n'ont pas d'eau potable. L'eau potable est importante pour nous et nos enfants mais la conservation de notre identité culturelle est meilleure. Dans tous les cas, tout le monde sur terre est appelé à mourir mais en partant, préserve ta culture pour ta descendance; donc nous sommes là». Ces propos montrent le désaccord des populations du cordon littoral depuis des décennies, à effectuer tout déplacement, qui pourrait les éloigner de leur héritage et de leur identité culturelle auxquels elles sont fortement attachées. Pour les populations du cordon littoral, quitter cet espace, c'est quitter : leurs origines, leurs dieux, leurs ancêtres, leur sable et leur mer avec lesquels elles ont un lien très étroit. Malgré la dégradation de leur espace par l'érosion côtière et la présence 
constante de maladies hydriques telles que : la fièvre typhoïde, la diarrhée et le choléra, rester sur leur terre demeure essentiel pour les Avikam du cordon littoral.

\section{2-2. Les contraintes environnementales du manque d'eau potable au sein des villages Avikam du cordon littoral de Grand-Lahou}

Au sein des villages Avikam du cordon littoral de Grand-Lahou, il existe un ensemble de sous-contraintes environnementales qui entravent l'accès à l'eau potable. Ce sont : l'enclavement de la zone, la proximité des villages des sources naturelles d'eau, l'absence de voie terrestre et l'érosion côtière.

\section{2-2-1. L'enclavement de la zone}

L'enclavement caractérise la fermeture ou l'isolement d'un espace. Le cordon littoral est un espace situé entre la lagune et l'océan Atlantique. Son positionnement fait qu'il n'est pas accessible par voie terrestre. À la question de savoir pourquoi le cordon littoral manque toujours d'eau potable, un enquêté affirme : "Madame voyez-vous que le cordon littoral est un endroit très enclavé coupé du reste du pays! Ils sont encore à l'époque préhistorique. Concernant le manque d'eau potable, les structures en charge de l'eau ne savent même pas qu'ils existent bien que ces villages regorgent d'une très grande population. Quand un endroit est très enclavé, il ne peut bénéficier d'un quelconque développement ». De ces propos, il ressort que le cordon est un espace trop enclavé. Cet enclavement entraîne une méconnaissance réciproque entre les structures en charge de l'eau potable et les populations rurales et une sorte de rétractation des populations au point de ne mener aucune action véritable qui pourrait aboutir à un changement hydrique. L'isolement de cette zone fait qu'elle est moins privilégiée quand il s'agit de projets de développement par les structures étatiques qui préfèrent des espaces désenclavés. L'enclavement de cette zone bloque toute promptitude des structures étatiques ivoiriennes à solutionner les problèmes d'accès à l'eau potable que vivent les populations depuis plusieurs décennies.

\section{2-2-2. La proximité des villages des sources naturelles d'eau}

Elle consiste pour les villages à être trop proches des sources d'eau que sont la lagune, le fleuve Bandama et l'océan Atlantique (photo1). Malgré ce vaste patrimoine hydrique, les villages ne possèdent pas d'ouvrages hydrauliques. Pour expliquer ce manque d'eau potable, un responsable administratif affirme : "Les villages du cordon sont très proches de l'eau (la mer et la lagune), ce qui fait que dans un premier temps l'eau est salée. Ensuite tu creuses juste 1 à $2 \mathrm{~m}$ tu as de l'eau, l'eau est en sub-surface. De plus, la mer d'ici est très agitée au point où elle détruit tout sur son passage surtout 
l'acier donc elle peut détruire les pompes sans problème. C'est tous ces éléments qui font que l'accès à l'eau potable là-bas est très difficile». Le positionnement de cette bande de terre fait que l'eau souterraine est en finesurface et possède une forte concentration en chlorure de sodium $(\mathrm{NaCl})$. Or l'installation d'ouvrages hydrauliques nécessite une profondeur variant de (10 à $60 \mathrm{~m}$ et plus) pour une installation durable et une eau souterraine non saline (Tonga, 2018). La proximité des villages à l'océan Atlantique entraîne des destructions de résidences, de fer et d'acier par la forte brise de mer. Cette situation contraint ainsi la plupart des populations à faire des constructions en bois.

Retenons de cette partie que la proximité des villages des sources naturelles d'eau a provoqué l'intrusion de l'océan Atlantique dans l'eau souterraine en la modifiant en une eau saline impropice à l'installation d'ouvrages hydrauliques qui nécessitent une eau souterraine potable.

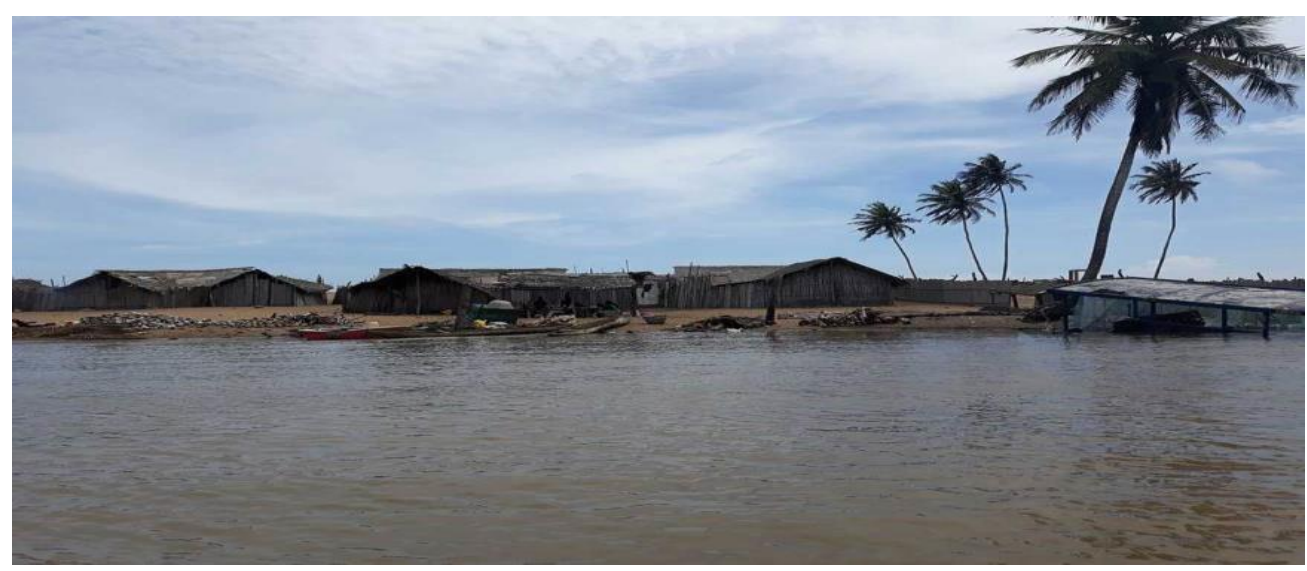

Photo 1 : La proximité des villages des sources d'eaux naturelles

Source : Notre archive 2015

\section{2-2-3. L'absence de voie terrestre}

Le cordon littoral est un espace social positionné entre la lagune et l'océan Atlantique. On n'y a accès uniquement que par voie lagunaire ou maritime. Les moyens pour y accéder sont : les hors-bords (pirogue à moteur), le bac ou les pirogues artisanales (photo2). 


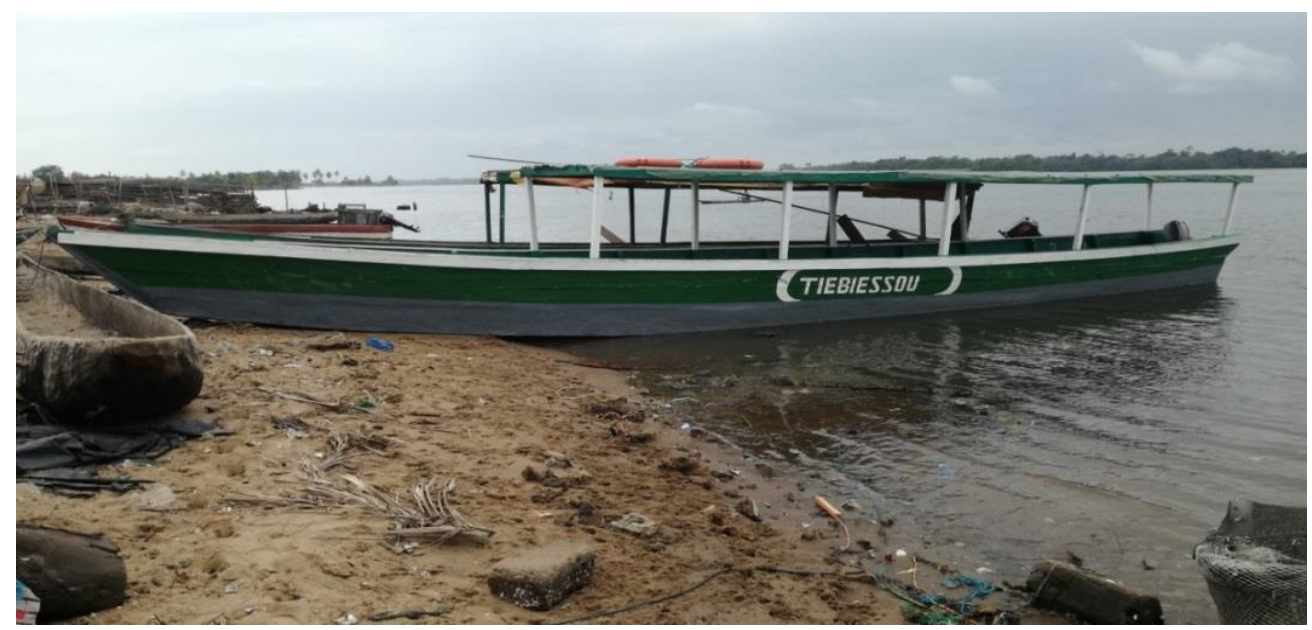

Photo 2 : Hors-bord pour accéder au cordon littoral

Source : Notre archive 2015

L'absence de voie routière pour avoir accès au cordon littoral est un fait, évoqué par la plupart des autorités départementales et régionales quant au transport du matériel hydraulique. À cet effet, le propos d'un enquêté vient exposer la difficulté pour accéder au cordon littoral de Grand-Lahou. "C'est vraiment difficile de se rendre sur le cordon littoral, le bac se limite sur la grande île. Quant aux hors-bords, ils ne peuvent pas transporter un matériel hydraulique pour un HVA donc c'est ce qui est le problème ici ». On constate qu'il n'y a pas de voie terrestre pour accéder au cordon littoral de Grand-Lahou comme le traduit ce propos. Alors que, la route permet les transactions, les échanges de biens, de services et le transport de matériels pouvant servir à la construction de plusieurs infrastructures modernes telles que : les ouvrages hydrauliques, sanitaires et électriques. Or, dans le cas des villages du cordon littoral, l'absence de voie terrestre limite toute possibilité à l'obtention d'HV, d'HVA et de châteaux; à cela s'ajoute l'absence d'électricité qui est indispensable à l'installation d'ouvrages hydrauliques tels que : les HVA et les châteaux.

\section{2-2-4. L'érosion côtière}

L'une des principales contraintes environnementales mentionnées tout au long de cette étude par l'ensemble des parties prenantes (structures étatiques et populations rurales) est l'érosion côtière. L'érosion côtière est une perte de la terre au profit de la mer, elle a de graves conséquences socio-économiques et environnementales. À ce sujet, un enquêté déclare ceci : «Normalement les villages du cordon ne devraient pas manquer d'infrastructures modernes telles que : l'eau potable, l'électricité, les plages, les espaces paradisiaques etc. Ce lieu devait être un lieu touristique qui drainerait beaucoup de monde 
comme Bassam. Mais c'est l'érosion côtière depuis les années 1970 qui a mis le cordon dans cette situation. L'érosion côtière a détruit l'ancienne ville, les bâtiments coloniaux et tout ce qu'elle renfermait. Actuellement même certaines tombes sont englouties allez-y à Lahou-Kpanda, vous verrez que la mer est proche des habitations elle avance à grands pas en détruisant tout sur son passage ». À travers ces propos, on peut affirmer que l'érosion a plongé le cordon dans un retard socio-économique et culturel tout en inondant l'ancienne ville de Grand-Lahou et tous les sites historiques dont elle regorgeait après la colonisation qui faisait de Grand-Lahou un espace attractif. L'érosion côtière a décimé toutes les plantations de cocoteraies et même une partie du cimetière qui représente un lieu sacré protégé où reposent les ancêtres protecteurs. La présence de l'érosion côtière au sein du cordon littoral menace la stabilité des villages et demeure le principal obstacle à la réalisation d'infrastructures modernes (l'eau potable, électricité etc.).

\section{Discussion}

La question de la disponibilité des populations en eau potable est un problème de souveraineté nationale à travers le monde. Il semble que cette situation de manque d'eau potable abordée dans la présente recherche est récurrente dans certains milieux et caractérise la zone de recherche. Pour comprendre les déterminants de cette problématique dans les villages Avikam du cordon littoral, la présente étude a essayé d'aborder les pistes suivantes :

\section{3-1. Les contraintes sociales du manque d'eau potable}

Au sein des villages Avikam, les structures étatiques développent des idéologies telles que : la disparition du cordon et le refus des populations de changer de site pour expliquer la vulnérabilité hydrique existante. Cependant, ces idéologies dissimulent des contraintes sociales que sont : le refus de l'État d'investir à perte et l'attachement des populations à leur patrimoine ancestral qui entravent l'accès à l'eau potable. Ces résultats corroborent celui de Kam (1998) qui estime qu'à Tanda, en dépit de l'existence d'ouvrages hydrauliques, les populations continuent de s'approvisionner en eau par le biais des sources traditionnelles. Les populations justifient cet abandon des forages par le fait qu'ils ont été réalisés près des forêts sacrées, classées et des cimetières qui sont des lieux de cultes et d'adorations par excellence. Pour elles, fréquenter donc ces endroits serait profaner l'esprit des ancêtres fondateurs du village. La réalisation de ces ouvrages ne tient pas compte des réalités culturelles de ces populations d'où leur abandon au profit des sources traditionnelles. Cette idée sera renchérie par Farah (2003) qui pense que l'échec de nombreux projets de développement en Afrique en général et dans le domaine de l'accès à l'eau potable en particulier est dû à la non prise en compte d'éléments culturels des sociétés où les projets sont implantés. Par 
ailleurs, l'auteur estime que les décisions sur les sites des projets sont prises au sommet par des personnes étrangères au milieu et qui ne maîtrisent pas trop les us et coutumes de la collectivité qui est censée en bénéficier. Cette réalité explique le manque d'eau potable au sein de certains milieux ruraux. Il ressort de ces études que la non prise en compte des aspects culturels est une contrainte sociale qui peut occasionner le manque d'eau potable en milieu rural. Berton (2010) quant à elle aborde l'inorganisation des structures en charge de l'accès à l'eau potable comme contrainte sociale du manque d'eau potable. Elle affirme que plusieurs programmes et projets ont été initiés par le Congo afin de résoudre les problèmes d'accès à l'eau potable malgré le manque de schéma directeur des services de l'hydraulique. Après la remise des ouvrages aux bénéficiaires sans disposition sur la prise en charge des points d'eau par les utilisateurs, la plupart des pompes installées ne sont plus fonctionnelles. Ce qui n'as pas permis de freiner la dégradation de la situation sanitaire des populations, due aux maladies hydriques et une faible proportion des populations rurales ayant l'accès à l'eau potable. Selon l'auteur, malgré l'importance de ses potentialités hydriques, la République Populaire du Congo est en situation de pénurie d'eau potable tant en villes qu'en milieu rural. Les problèmes d'eau et d'assainissement touchent non seulement les paysans congolais, mais aussi ceux d'autres pays en développement. L'analyse de ces différents articles révèle l'existence d'ouvrages hydrauliques qui ont été soit abandonnés dans certains milieux en raison de la non prise en compte de certains aspects culturels dans leur construction ou soit dégradés par manque de politique hydrique pouvant permettre leur pérennisation. Le cas des villages Avikam est particulier puisqu'en dépit de l'attachement des populations à leur identité culturelle et à la terre de leurs ancêtres, il y a le refus des structures étatiques ivoiriennes à faire des investissements sans rentabilité sur un espace impacté par des contraintes environnementales.

\section{3-2. Les contraintes environnementales qui entravent le manque d'eau potable}

Le manque de l'eau potable au sein des villages Avikam du cordon littoral de Grand-Lahou peut s'expliquer par la présence d'un certain nombre de contraintes environnementales que sont: l'enclavement de la zone, l'absence de voie terrestre, la proximité des villages des sources naturelles d'eau et l'érosion côtière qui font obstacle à tout processus d'installation d'ouvrages hydrauliques. Ces résultats sont similaires à celui réalisé par Dégbey(2008) dans l'arrondissement de Toviklin au Bénin. Dans son article, l'auteur fait l'état de ce que les problèmes d'approvisionnements en eau potable que connaît la commune de Toviklin, sont dues à la diversité de nature géologique de la zone ; à la difficulté du plan hydrogéologique ; à la limitation de la nappe de la région et à certaines profondeurs du sous-sol. L'ensemble de 
ces irrégularités au niveau du sous-sol peut expliquer le tarissement précoce de plusieurs points d'eau traditionnels dans la région et la difficulté à obtenir de l'eau potable. Ces irrégularités au niveau du sous-sol constituent des contraintes environnementales qui entraînent les problèmes d'accès à l'eau potable. Cette réalité est également mentionnée dans les travaux de Djedji (2011) réalisés dans la commune de Lalo au Benin ; il affirme que les problèmes d'accès à l'eau potable sont imputables aux accidents tectoniques du sol qui nécessitent plus de moyens financiers et de disponibilité. À cette première difficulté, s'ajoute l'impraticabilité des pistes des localités de cette partie de la commune de Lalo, ce qui rend l'implantation d'ouvrages hydrauliques difficile, voire impossible. Foe (2019) quant à lui, aborde l'impact des changements climatiques sur l'accès à l'eau potable. Selon l'auteur, les effets néfastes des changements climatiques sont perceptibles à travers le tarissement des points d'eau et les inondations des espaces, des déchets alimentaires, industriels et fécaux qui par la suite se déversent dans les cours d'eau en polluant les eaux souterraines et les eaux dites potables. Les changements climatiques influent sur la quantité et la qualité de l'eau. De ce qui précède, on peut affirmer que les contraintes environnementales sont des réalités naturelles qui rendent l'accès à l'eau potable dans certains milieux, difficile voire impossible.

En somme, on peut retenir que l'existence de contraintes socioenvironnementales dans un espace entraîne le manque d'eau potable, qui a des répercussions socio-économiques et sanitaires néfastes sur les populations.

\section{Conclusion}

La présente étude a permis de mettre en exergue le manque d'eau potable en corrélation avec des contraintes socio-environnementales au sein des villages Avikam du cordon littoral. Au terme de ce travail, on peut affirmer qu'il existe deux (2) catégories de contraintes qui font obstacle à l'accès à l'eau potable au sein desdits villages dans le département de Grand-Lahou. La première catégorie appelée contraintes sociales est constituée : du refus de l'État d'investir à perte et l'attachement des populations à leur terroir. Quant à la deuxième catégorie dénommée contraintes environnementales, elle comprend l'enclavement de la zone, la proximité des villages aux sources naturelles d'eau, l'absence de voie terrestre et l'érosion côtière. L'ensemble de ces contraintes rend l'accès à l'eau potable difficile, voire impossible au sein des villages Avikam du cordon littoral de Grand-Lahou. Malgré la présence de ces contraintes, l'État peut mettre en place des stratégies telles que

$\checkmark$ le rapprochement des structures étatiques en charge de l'eau aux populations rurales afin de faciliter les échanges et les sensibilisations ; 
$\checkmark$ la sensibilisation pratique de la population sur la nécessité d'entretenir les points d'eau existants dans les différents villages ;

$\checkmark$ la sensibilisation sur comment conserver et faire bouillir l'eau de boisson avant consommation ;

$\checkmark$ la transformation des puits traditionnels en puits modernes ;

$\checkmark$ la distribution gratuite des produits (javel, pastille de chlore, les comprimés etc.) de façon mensuelle pour éliminer tous les microorganismes pouvant exposer à des maladies hydriques.

L'ensemble de ces stratégies va réduire les maladies hydriques et permettre aux populations rurales d'avoir accès à une eau potable, propre et salubre comme l'indique la convention de l'assemblée générale de l'ONU (ONU, 2010).

\section{References :}

1. Albarello L., Digneffe F., Hiemaux J.P., Maroy C., Ruquoy, D. \& Pierre de S.G., (1995). Pratiques et méthodes de recherche en sciences sociales, Armand collin, Paris, pp. 112-139.

2. Berton Y.O., (2010). L'approvisionnement en eau des populations rurales au Congo-Brazzaville, Les Cahiers d'Outre-Mer [En ligne], 249 | Janvier-Mars 2010, mis en ligne le 01 janvier 2013, consulté le 07 février 2021. URL:

http://journals.openedition.org/com/5838; DOI: https://doi.org/10.4000/com.5838. Pp.7-30.

3. Dadi $\mathrm{P},(2020)$ Carte de localisation des 4 villages du cordon littoral Grand-Lahou, thèse de doctorat uniqe en sociologie,source BNED 2014, 74p.

4. Dégbey D., (2008). Problèmes d'approvisionnement en eau potable dans l'arrondissement de Toviklin, Université d'Abomey CalaviMaîtrise en Géographie et aménagement du territoire, En ligne www.memoireoneline.com, pp. 26-70.

5. Diabagaté A., Konan G.H. \& Koffi A., (2016). Stratégies d'approvisionnement en eau potable dans l'agglomération d'Abidjan (Côte d'Ivoire), Géo-Eco-Trop., 4 : 345-360. http :jjwww.geoecotrop.be.

6. Djedji T.M., (2011). Gestion et contraintes géographiques des points d'eau communautaires en milieu rural dans la commune de Lalo Université d'Abomey Calavi (UAC)-Maîtrise. En ligne www.memoireoneline.com, pp 23-59.

7. Farah F., (2003). Population, traditions and développement in western Côte-d'Ivoire. In une science pour l'humanité. Centre de recherche pour le développement international, ID 39209, Ottawa (Canada). 
8. Foe B.J., (2019). Changement climatique, accès à l'eau potable et santé publique: entre réalités et perspectives en Afrique. Institut de Recherche Socio Anthropologiques (IRSA/UCAC), Article académique, pp. 6-9.

9. Hamaji Magazine, (2016). Grand-Lahou, entre deux flots, une fenêtre ouverte sur l'histoire, en ligne.www.hamajimagazine.com, $\mathrm{p} 1$.

10. INS, (2014). Recensement Général de la Population et de l'Habitat, Résultat globaux, Archive des données du département de GrandLahou, 22 p.

11. Kam O., (1998). Problématique de la gestion des infrastructures d'hydrauliques dans les projets d'approvisionnement du milieu rural en eau potable, Ed. Université Félix-Houphouët-Boigny-Abidjan. In www.revue-socioloique.org. Sites. Defaut.files, pp 5-12.

12. Marteau M., (2017). $1^{\text {er }}$ projet d'adduction d'eau potable d'envergure en côte d'ivoire ; en ligne www.vergnet-hydro.com, $\mathrm{p} 1$

13. Ministère de l'hydraulique, (2019). Projets réalisés de 2011 à 2019, en ligne hydraulique. Gouv.ci, pp. 9-12.

14. Nogbou J., (2018). Rapport bilan de la SODECI de Grand-Lahou, 25 p.

15. ODD, (2015). Les 17 Objectifs de développement durable et leurs 169 cibles, en ligne www globalcompact-France.org-documents-les 17. 7 p.

16. ONEP, (2017). Présentation du projet eau pour tous, en ligne, $w w w$ onep.ci présentation-programme-eau, $\mathrm{p} 1$.

17. ONU, (2010). L'Assemblée 《 reconnaît » le droit à l'eau potable comme un droit fondamental, www.un.org. Press.AG1097.doc.htm, p3.

18. Tchagba L., (2019). Programme social du Gouvernement volet hydraulique, en ligne psgouv.ci, pl.

19. Tonga T., (2018). Plan d'installation des ouvrages hydrauliques, $50 \mathrm{p}$. 